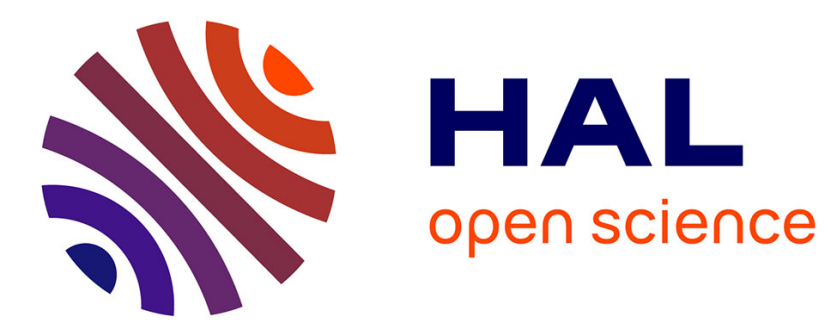

\title{
Spin transition in SrFeO2 under Pressure by X-ray spectroscopy
}

\author{
J.-P Rueff, V. Balédent, K Higashi, H Kageyama
}

\section{To cite this version:}

J.-P Rueff, V. Balédent, K Higashi, H Kageyama. Spin transition in SrFeO2 under Pressure by X-ray spectroscopy. 2020. hal-02908538

\section{HAL Id: hal-02908538 \\ https://hal.science/hal-02908538}

Preprint submitted on 29 Jul 2020

HAL is a multi-disciplinary open access archive for the deposit and dissemination of scientific research documents, whether they are published or not. The documents may come from teaching and research institutions in France or abroad, or from public or private research centers.
L'archive ouverte pluridisciplinaire HAL, est destinée au dépôt et à la diffusion de documents scientifiques de niveau recherche, publiés ou non, émanant des établissements d'enseignement et de recherche français ou étrangers, des laboratoires publics ou privés. 


\title{
Spin transition in $\mathrm{SrFeO}_{2}$ under Pressure by X-ray spectroscopy
}

\author{
J.-P. Rueff \\ Synchrotron SOLEIL, L'Orme des Merisiers, BP 48 St Aubin, 91192 Gif-sur-Yvette, France* and \\ Laboratoire de Chimie Physique-Matière et Rayonnement, \\ Sorbonne Université, CNRS, 75005 Paris, France; \\ V. Balédent \\ Laboratoire de Physique des Solides, CNRS, Université Paris-Sud, \\ Université Paris-Saclay 91405 Orsay Cedex, France \\ K. Higashi and H. Kageyama \\ Department of Chemistry, Graduate School of Science, \\ Kyoto University, Sakyo, Kyoto 606-8502, Japan,
}

(Dated: July 29, 2020)

\begin{abstract}
We have investigated the local Fe electronic changes in the square planar $\mathrm{Fe}(\mathrm{II})$ compound $\mathrm{SrFeO}_{2}$ by x-ray emission (XES) and x-ray absorption spectroscopy (XAS) through the pressure-induced spin transition up to $60 \mathrm{GPa}$. The analysis of Fe $K \beta$ XES confirms the transition under pressure from a high spin to intermediate spin state at $P_{c}=43 \mathrm{GPa}$ while XAS at the Fe K-edge reveals the spectral signatures of the lattice compression under pressure. Simulations of the XAS spectra point to significant changes in Fe $p$ - $d$ states across $P_{c}$ through the hybridization with the $\mathrm{O} p$ states eventually leading to the magnetic instability.
\end{abstract}

\section{INTRODUCTION}

Spin transitions are widely observed, important phenomena with potential use for ultrafast switches, data storage devices, and optical displays [1]. Spin transitions are generally found in octahedrally coordinated $3 d$ transition metal (TM) ions such as oxides [2, 3], metal-organic complexes $[4,5]$ or molecular magnets $[6,7]$. In the presence of the crystal electric field in $O_{h}$ symmetry, the $3 d$ orbitals are split into $t_{2 g}$ and $e_{g}$ orbitals, whose energy gap depends on the crystal field strength. The filling of these orbitals by the $3 d$ electrons governs the TM spin state and is determined by the competition between the crystal field strength and the intra-atomic exchange interaction. External perturbations such as temperature, pressure, magnetic field or light pulses can modify the relative strength of these interactions, eventually inducing a spin state transition. The stability of the spin state has profound consequences on the materials behavior as it affects its structural stability, magnetic ordering and transport properties, which explains its broad interest in condensed matter physics, molecular chemistry or mineralogy.

Recently, Kawakami et al. [8] have reported a spin state transition in the $\mathrm{Fe}(\mathrm{II}) \mathrm{SrFeO}_{2}$ compound under pressure. As illustrated in Fig. 1, the structure of $\mathrm{SrFeO}_{2}$ consists of $\mathrm{FeO}_{2}$ 2D layers separated by $\mathrm{Sr}$ atoms [9] with Fe sitting in a square planar site with $D_{4 h}$ symmetry. Using Mössbauer spectroscopy, x-ray diffraction, and transport measurements, the authors showed that the Fe spin changes from high spin (HS, $S=2$ ) to intermediate spin state (IS, $S=1$ ) state around $P_{c}=33$

\footnotetext{
* jean-pascal.rueff@synchrotron-soleil.fr
}

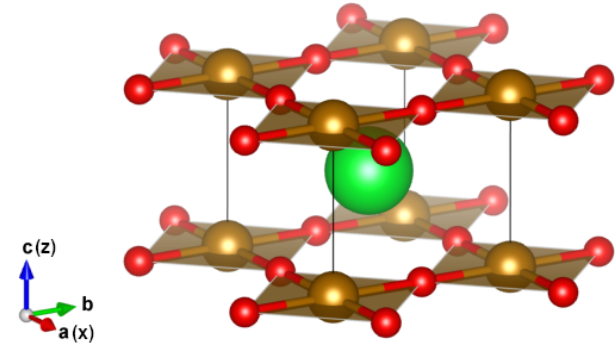

FIG. 1. $\mathrm{SrFeO}_{2}$ structure in the $P 4 / \mathrm{mmm}$ space group highlighting the square planar environment of $\mathrm{Fe}$ (yellow balls) surrounding by four $\mathrm{O}$ atoms (red balls). The Sr atom (green ball) lies in the interplanes region. The local $x$ and $z$ axes are aligned with the $a$ and $c$ directions.

GPa ; the spin transition is accompanied by a transition from an antiferromagnetic (AFM) insulating state to likely a ferromagnetic (FM) half-metallic state ; at the same time, an anomalous drop of the lattice parameters is found at $P_{c}$. This makes $\mathrm{SrFeO}_{2}$ the first example of a four-fold coordinated TM compound undergoing a spin transition. Similar results were more recently observed in the structurally related two-legged spin ladder compound $\mathrm{Sr}_{3} \mathrm{Fe}_{2} \mathrm{O}_{5}$ where $\mathrm{Fe}$ also occupies in a square planar site Kageyama et al. [10]. Akin to $\mathrm{SrFeO}_{2}, \mathrm{Sr}_{3} \mathrm{Fe}_{2} \mathrm{O}_{5}$ undergoes a pressure-induced spin state transition, an insulator to metal transition, and AFM to FM transition with a magnetic transition at $34 \mathrm{GPa}$ [11].

In this article, we provide a detailed view at the local Fe level of the transition mechanism and change of the electronic structure under pressure in $\mathrm{SrFeO}_{2}$ as observed by X-ray Emission Spectroscopy (XES) at the Fe K $\beta$ emission line and X-ray Absorption Spectroscopy (XAS) at the Fe K-edge [12]. Thanks to the resonant excita- 
tion, x-ray spectroscopy in the hard x-ray range is an effective element and orbital selective probe of the electronic properties in transition metal compounds, while being compatible with high pressure environment. The results confirm the spin state transition from HS to IS state at high pressure and demonstrate that significant changes in Fe $p$ - $d$ states occur across $P_{c}$ through the hybridization with the $\mathrm{O} p$ states eventually leading to the magnetic instability.

\section{RESULTS AND DISCUSSION}

\section{A. Methodology}

$\mathrm{SrFeO}_{2}$ sample was synthesized at Kyoto University as described in Ref. [8]. The sample was prepared as powder and loaded in a membrane driven diamond anvil cell (DAC) with $300 \mu \mathrm{m}$ culet size. We used Rh as gasket material with a $150 \mu \mathrm{m}$ hole. The in-situ pressure was estimated from the photo luminescence of ruby chips that were placed in the sample chamber. We performed two successive loadings of the pressure cell to cover the entire pressure range with two different pressure transmitting medium : Neon gas (for the 0-60 GPa pressure range) and 1:4 methanol-ethanol mixture (for 50-60 GPa). The two datasets were eventually combined as no visible difference could be detected between them, either in the XAS and XES spectral shape or behavior under pressure.

The experiment has been carried out the GALAXIES x-ray spectroscopy beamline at Synchrotron SOLEIL [13]. The incident photons were monochromatized by a cryogenically cooled $\mathrm{Si}(111)$ double crystal monochromator and focused onto the sample by a toroidal mirror in a $30 \mu \mathrm{m} \times 80 \mu \mathrm{m}$ spot. The Fe $\mathrm{K} \beta$ spectra were measured on the beamline XES spectrometer in transmission geometry through the DAC at a scattering angle of $15^{\circ}$. The spectrometer was equipped with a $\mathrm{Si}(531) 1-$ $\mathrm{m}$ spherically bent crystal analyzer operated around the Bragg angle of $73.1^{\circ}$ and a silicon drift diode (SDD) as detector. The XES spectra were acquired in a continuous scanning mode by sweeping the emission energy from 7020 to $7080 \mathrm{eV}$ at fixed incident photon energy of 9000 $\mathrm{eV}$. This energy was found to yield the the strongest emission intensity in the DAC. At some selected pressure, we also acquired high-resolution XAS spectra in the partial fluorescence yield (PFY) mode by setting the spectrometer emission energy at the maximum of the $\mathrm{K} \beta$ emission line while sweeping the incident photon energy through the Fe K-edge. This method - also known as high energy resolution fluorescence detected (HERFD) XAS - yields the absorption spectrum with improved intrinsic resolution with respect to conventional XAS as the core-hole lifetime broadening has been partly removed owing to the second order scattering process [14].

\section{B. Results}

\section{XES}

The Fe K $\beta$ XES spectra are shown in Fig.2 as a function of pressure $P$. To emphasize the spectral changes in the figure, the spectra were normalized to the maximum intensity and aligned in energy. The spectra are composed of a large intensity peak and a weaker, low energy satellite respectively referred to as $\mathrm{K} \beta_{1,3}$ and $\mathrm{K} \beta^{\prime}$. As it is now well established, the $\mathrm{K} \beta$ spectral shape and specially the satellite region is sensitive to the magnitude $S$ of the local spin state of the $3 d$ ion $[15,16]$.

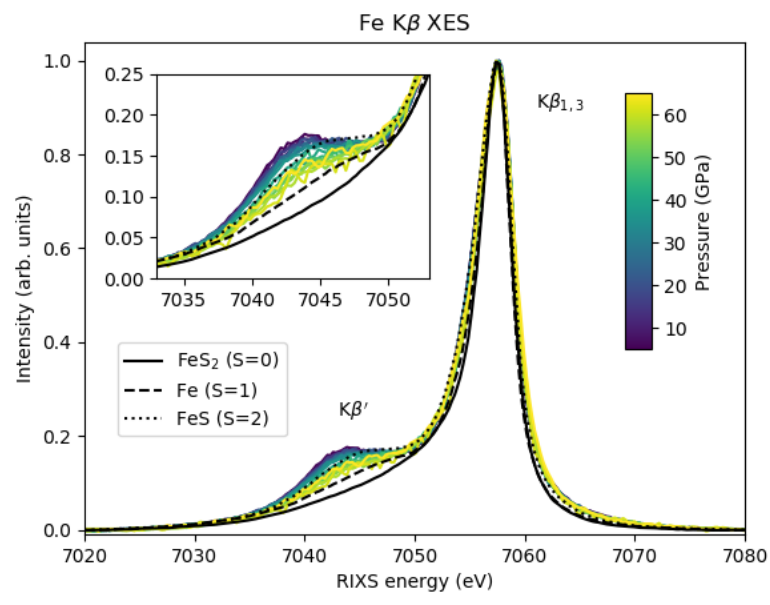

FIG. 2. Fe K $\beta$ XES spectra as a function pressure; the inset shows a zoom of the satelite $\left(\mathrm{K} \beta^{\prime}\right)$ region. References spectra representative of $S=0,1,2$ spin state and measured in $\mathrm{FeS}_{2}$, Fe metal and FeS are shows with black solid, dashed and dotted lines from [17]

To first assess the nature of the high pressure phase, we compare in Fig. 2 the emission spectra in $\mathrm{SrFeO}_{2}$ with spectra measured in reference compounds borrowed from Ref. [17], the $\mathrm{Fe}(\mathrm{II})$ compounds $\mathrm{FeS}$ and $\mathrm{FeS}_{2}$ with nominal spin states $S=2$ and $S=0$ and Fe metal with nominal $S=1$. While $\mathrm{FeS}_{2} \mathrm{XES}$ spectrum shows no distinct $\mathrm{K} \beta^{\prime}$ feature, $\mathrm{Fe}$ metal exhibits is weak satellite which seems very comparable to $\mathrm{SrFeO}_{2}$ high pressure spectra. This qualitatively confirms the intermediate spin state $(S=1)$ assignment of the high pressure phase of $\mathrm{SrFeO}_{2}$. The low pressure spectra compares well with that of the $S=2$ compound FeS although the satellite intensity is somewhat higher in $\mathrm{SrFeO}_{2}$, likely due to the different $\mathrm{Fe}$ environment and degree of covalency.

The pressure dependence of the local spin moment $S$ can be more quantitatively estimated by means of the Integral Absolute Difference (IAD) method which has been developed earlier [4, 18]. Following Vankó et al. [4], the IAD values were computed by integrating the spectral difference $|\operatorname{XES}(P)-\operatorname{XES}(P=0)|$ after normalizing the spectral intensity to the total area and align- 


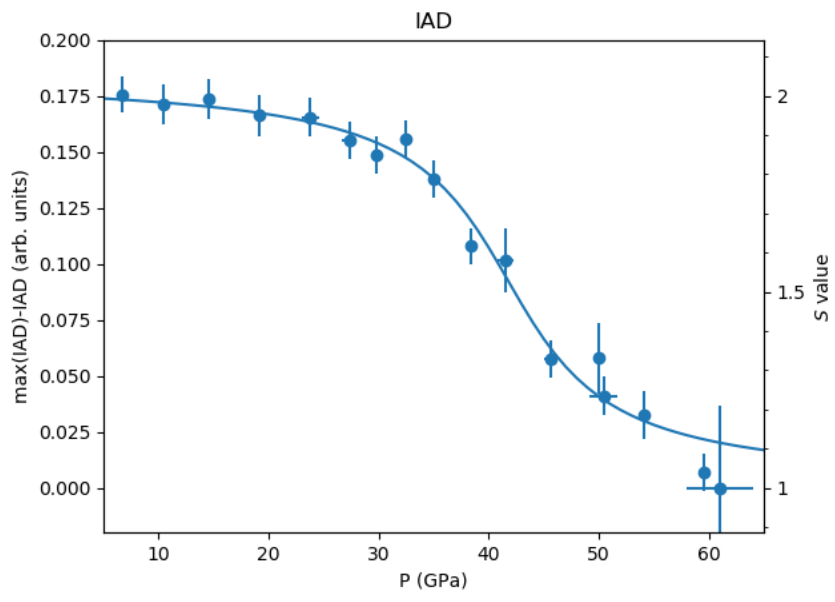

FIG. 3. Computed IAD as a function of pressure (circles) and arctan fit (solid line); the IAD is converted onto a indicative spin scale (right axis)

ing the spectra to the center of gravity. Figure 3 shows the IAD change (here expressed as max (IAD) - IAD $(P)$ ) as a function of pressure. This pressure dependence is very comparable to the one obtained by other methods of evaluating $S$, including first moment analysis or satellite intensity, demonstrating its robustness. Using the reference spectra of Figure 3, we have the converted the IAD values onto an indicative spin scale represented by the right axis in Figure 3. The IAD analysis confirms the pressure-induced transition form HS to IS states. We can obtain a rough estimate of the transition pressure $P_{c}$ by fitting the IAD dependence with an arctan function :

$$
H / \pi\left(\pi-\arctan \left(2 / w\left(P-P_{c}\right)+\pi / 2\right)\right)
$$

with $H$ the step height and $w$ the distribution width. The fit shown in Fig. 3 with solid line yields $P_{c}=42 \mathrm{GPa}$, a value somewhat higher than value of $33 \mathrm{GPa}$ reported by Kawakami et al. [8]. The width $w$ is estimated at 14 $\mathrm{GPa}$, which could be due to pressure gradient and phase mixing.

\section{2. $X A S$}

We now turn to the XAS results which provides a local view of structural change across the pressure induced spin transition. The Fe K-ege X-ray absorption spectra were measured at two pressures across $P_{c}$ in the low pressure (LP) phase at $0 \mathrm{GPa}$ and high pressure (HP) phase at $45 \mathrm{GPa}$. Figure 4 (top panel) displays the two spectra on a relative energy scale $E-E_{0}$ with $E_{0}=7115.5 \mathrm{eV}$. The spectra were corrected for self-absorption. For the sake of comparison, the XAS spectra were normalized in intensity to an edge jump of unity and align in energy so that the flex point falls at $E_{0}$. The spectra are composed of an intense pre-edge feature $(0-5 \mathrm{eV})$ of mixed $p$ - $d$ char- acter (as explained bellow) followed by an intense edge jump (5-20 eV) related to the $p$ states due to dipolar transitions. The spectra undergo a drastic change with pressure marked by a shift of the pre-edge and post-edge features toward high energy along with a sizable broadening.
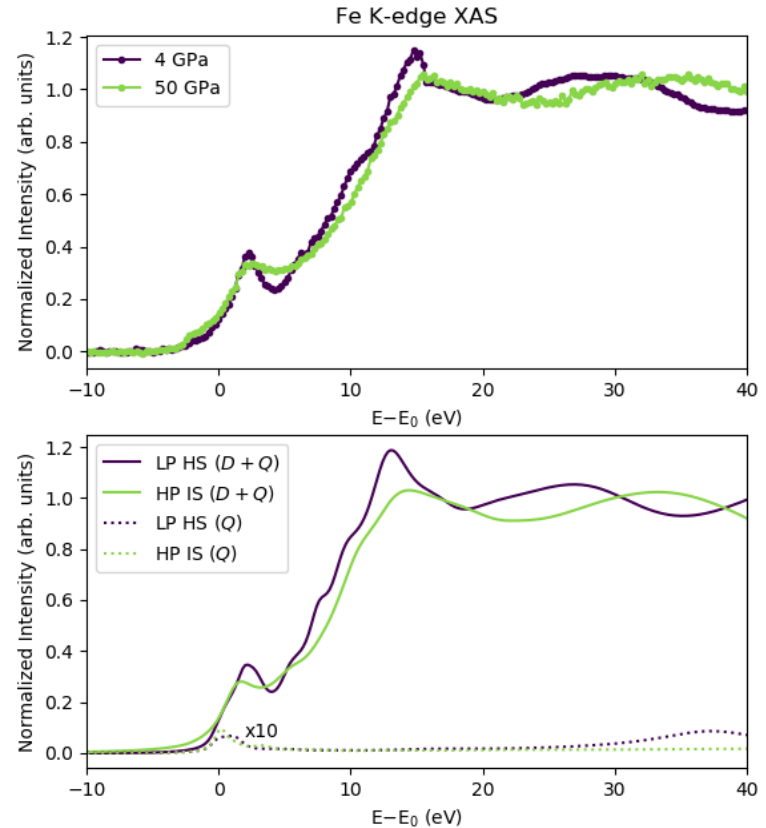

FIG. 4. (top panel) Experimental Fe K-edge XAS spectra in the low pressure (LP) and high pressure (HP) phases ; (bottom panel) Computed spectra using FDMNES in both LP and HP phases (solid lines) ; $D$ and $Q$ refer to the dipolar and quadrupolar contributions. For the sake of clarity, the latter (dashed lines) has been multiplied by 10 .

To get deeper insight in the spectral changes, the experimental spectra are compared to the first-principle calculations (Fig.4, bottom panel). The calculations were performed using the FDMNES code [19] on a $7 \AA$ radius atomic cluster within the $\mathrm{LDA}+\mathrm{U}$ approximation. The Hubbard parameter was set to $U=5 \mathrm{eV}$ for the LP phase in agreement with the previous works $[20-22]$ and $U=0$ $\mathrm{eV}$ for the HP phase following the vanishing $U$ value predicted at high pressure in Rahman et al. [20] and the observation of the gap closing around $50 \mathrm{GPa}[8]$. We used the structural parameters obtained at $0 \mathrm{GPa}(a=3.991$ $\AA, c=3.474 \AA)$ and $45 \mathrm{GPa}(a=3.744 \AA, c=3.002 \AA)$ obtained from Refs. $[8,9]$ within the same space group $P 4 / \mathrm{mmm}$ as illustrated in 1 . We forced Fe to adopt a $3 d^{6}$ electronic configuration with a high-spin (respectively intermediate spin) state at 0 (resp. 45) GPa. The computation was conducted using self-consistent, non muffin tin potentials and including spin-orbit effect and both dipolar $(D)$ and quadrupolar $(Q)$ contributions. To take into account the sharpening effect due to the PFY approach, 
the computed spectra were convolved by a Lorentzian with FWHM of $0.5 \mathrm{eV}$ (below the $1 s$ lifetime estimated at $1.3 \mathrm{eV}$ ).

The pressure induced experimental changes are well reproduced by the calculations confirming that the observed spectral difference are driven by changes in the Fe electronic structure upon lattice compression. The quadrupolar contribution shown as dashed line in Figure 5 appears to be of minute intensity and located in a narrow energy window around $E_{0}$. This demonstrates that the overall spectrum including the pre-edge region is dominated by the dipolar component related to the Fe $p$ states. To evaluate the change of the electronic structure, we show in Fig. 6 the projected density of states (pDOS) of the absorbing Fe atom at low and high pressures. Our computed pDOS differ from previously reported works $[8,20-22]$ as it takes into account the $1 s$ core-hole present in the XAS final state. Our purpose moreover is to focus on the changes observed in the empty $d$ and $p$ states which are probed by XAS. A comparison with Fig. 5 confirms that the XAS spectra mainly reflects the Fe unoccupied $p$-pDOS with some admixture of $d$ character in the pre-edge region near $E_{0}$. The pDOS further highlight the strong hybridization between the Fe $p$ and $d$ states.
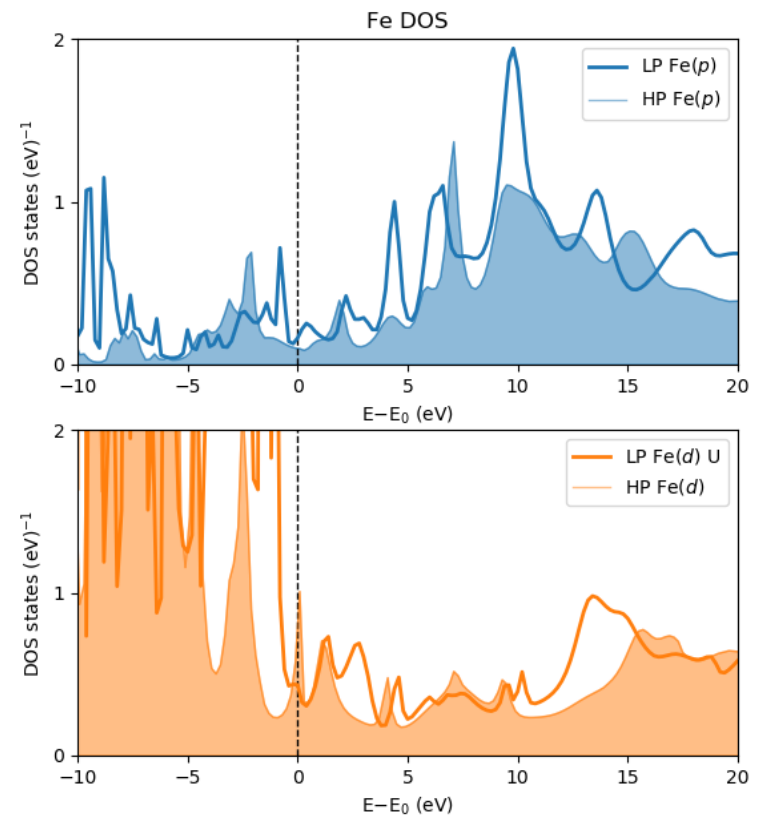

FIG. 5. (top panel) Computed Fe p-pDOS (top panel) and Fe $d$-pDOS (bottom panel) in the low pressure (thick line) and high pressure (thin filled line) phase

Figure 6 (top panel) shows the details of the Fe $d$ pDOS in the LP and HP phases. They are compared to the $\mathrm{O} p$-pDOS shown in the bottom panel. As reported in Ref. [8], there is a strong in-layer overlap between $\mathrm{O}$ $p$ states and $\mathrm{Fe} d_{x^{2}-y^{2}}$ states which appears prominent in the $0-4 \mathrm{eV}$ relative energy range. In the high pressure phase, the population of Fe $d_{x^{2}-y^{2}}$ empty pDOS increases as revealed by additional empty states around 1 and 3 $\mathrm{eV}$, which is reflected in the HP $\mathrm{O} p$ pDOS. This inlayer electronic instability is believed to drive the HS to IS spin transition. It is consistent with the electronic transfer from $d_{x^{2}-y^{2}}$ to $d_{x z, y z}$ at the transition initially suggested by Kawakami et al. [8].
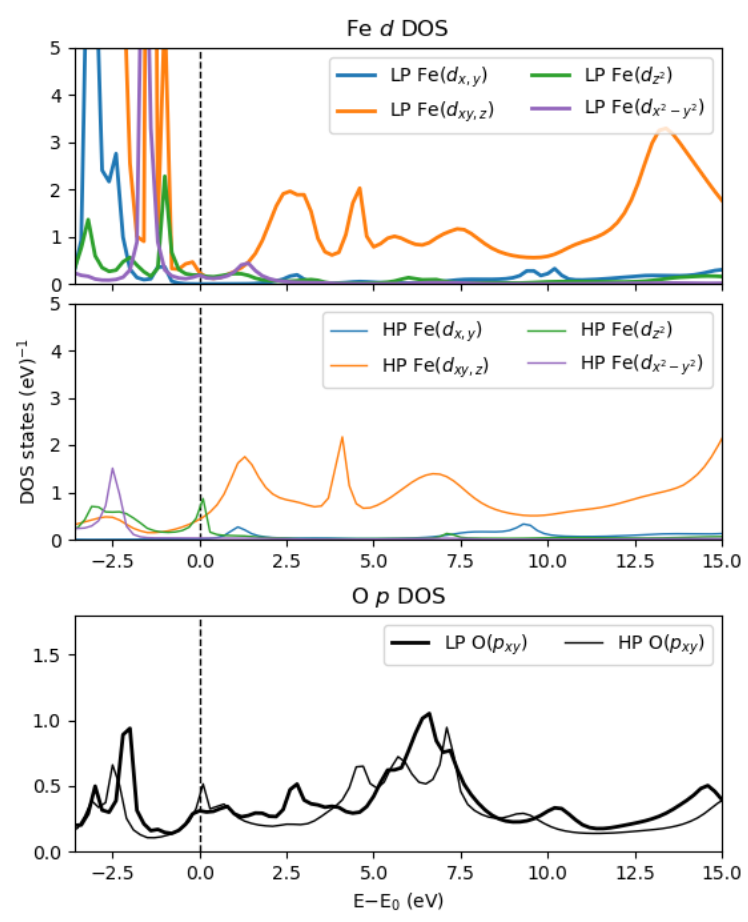

FIG. 6. Details of the Fe $d$-pDOS (two top panels) and O $p$-pDOS (bottom panel) in the low pressure (tick lines) and high pressure phase (thin lines)

We notice that while the AFM state in the LP phase is well established, the FM nature of HP phase is still debated. Because XES is a local probe, it cannot discriminate between FM or other magnetic structures. But other probes of the long range order such as X-ray magnetic dichroism at the Fe K-edge could be tempted in the future.

\section{CONCLUSIONS}

We have studied the pressure-induced spin transition in $\mathrm{SrFeO}_{2}$ by x-ray spectroscopy by combining $\mathrm{Fe} \mathrm{K} \beta$ XES and XAS at the Fe K-edge up to 60 GPa. The results provide a detailed picture of the $\mathrm{Fe}$ electronic changes across the magnetic transition from a local point of view. While Fe remains magnetic at $60 \mathrm{GPa}$, we can expect a second transition to another IS state or even a complete loss of magnetic moment at even higher pressure when the $d$-electrons become fully delocalized. This 
should motivate a continuation of this work towards the $100 \mathrm{GPa}$ range.

\section{ACKNOWLEDGMENTS}

We wish to acknowledge the SOLEIL Synchrotron for provision of beamtime (proposal 20181616). This work was supported by JSPS Core-to-Core Program (grant number: JPJSCCA20200004).
[1] J. Létard, P. Guionneau, and L. Goux-Capes, Towards spin crossover applications, in Topics in Current Chemistry (Springer, 2004) pp. 221-249.

[2] A. Mattila, J.-P. Rueff, J. Badro, G. Vankó, and A. Shukla, Metal-ligand interplay in strongly-correlated oxides:a parametrized phase diagram for pressure induced spin transitions, Phys. Rev. Lett. 98, 196404 (2007).

[3] M. Pasternak, R. Taylor, R. Jeanloz, X. Li, J. Nguyen, and C. McCammon, High pressure collapse of magnetism in $\mathrm{Fe}_{0.94} \mathrm{O}$ : Mössbauer spectroscopy beyond $100 \mathrm{GPa}$, Phys. Rev. Lett. 79, 5046 (1997).

[4] G. Vankó, T. Neisius, G. Molnar, F. Renz, S. Karpati, A. Shukla, and F. M. F. de Groot, Probing the 3d Spin Momentum with X-ray Emission Spectroscopy: The Case of Molecular-Spin Transitions, J. Phys. Chem. B 110, 11647 (2006).

[5] C. J. Pollock and S. DeBeer, Insights into the geometric and electronic structure of transition metal centers from valence-to-core x-ray emission spectroscopy, Accounts of Chemical Research 48, 2967 (2015).

[6] J.-D. Cafun, J. Lejeune, F. Baudelet, P. Dumas, J.-P. Itié, and A. Bleuzen, Room-temperature photoinduced electron transfer in a prussian blue analogue under hydrostatic pressure, Angewandte Chemie International Edition 51, 9146 (2012).

[7] D. Papanikolaou, S. Margadonna, W. Kosaka, S.-i. Ohkoshi, M. Brunelli, and K. Prassides, X-ray illumination induced fe(ii) spin crossover in the prussian blue analogue cesium iron hexacyanochromate, Journal of the American Chemical Society 128, 8358 (2006).

[8] T. Kawakami, Y. Tsujimoto, H. Kageyama, X.-Q. Chen, C. L. Fu, C. Tassel, A. Kitada, S. Suto, K. Hirama, Y. Sekiya, Y. Makino, T. Okada, T. Yagi, N. Hayashi, K. Yoshimura, S. Nasu, R. Podloucky, and M. Takano, Spin transition in a four-coordinate iron oxide, Nature Chemistry 1, 371 EP (2009).

[9] Y. Tsujimoto, C. Tassel, N. Hayashi, T. Watanabe, H. Kageyama, K. Yoshimura, M. Takano, M. Ceretti, C. Ritter, and W. Paulus, Infinite-layer iron oxide with a square-planar coordination, Nature 450, 1062 (2007).

[10] H. Kageyama, T. Watanabe, Y. Tsujimoto, A. Kitada, Y. Sumida, K. Kanamori, K. Yoshimura, N. Hayashi, S. Muranaka, M. Takano, M. Ceretti, W. Paulus, C. Ritter, and G. André, Spin-ladder iron oxide: Sr3fe2o5, Angewandte Chemie International Edition 47, 5740 (2008).
[11] T. Yamamoto, C. Tassel, Y. Kobayashi, T. Kawakami, T. Okada, T. Yagi, H. Yoshida, T. Kamatani, Y. Watanabe, T. Kikegawa, M. Takano, K. Yoshimura, and H. Kageyama, Pressure-induced structural, magnetic, and transport transitions in the two-legged ladder sr3fe2o5, Journal of the American Chemical Society 133, 6036 (2011).

[12] J.-P. Rueff, Spin-transitions in metal oxides, in SpinCrossover Materials (John Wiley and Sons, Ltd, 2013) Chap. 21, pp. 527-541.

[13] J.-P. Rueff, J. M. Ablett, D. Céolin, D. Prieur, T. Moreno, V. Balédent, B. Lassalle, J. E. Rault, M. Simon, and A. Shukla, The GALAXIES Beamline at SOLEIL Synchrotron: Inelastic X-ray Scattering and Photoelectron Spectroscopy in the Hard X-ray Range, J. Synchrotron Rad. 22, 175 (2015).

[14] K. Hämäläinen, D. P. Siddons, J. B. Hastings, and L. E. Berman, Elimination of the inner-shell lifetime broadening in x-ray absorption spectroscopy, Phys. Rev. Lett. 67, 2850 (1991).

[15] J.-P. Rueff, C.-C. Kao, V. V. Struzhkin, J. Badro, J. Shu, R. J. Hemley, and H. K. Mao, Pressure induced high-spin to low-spin transition in FeS evidenced by x-ray emission spectroscopy, Phys. Rev. Lett. 82, 3284 (1999).

[16] F. M. F. de Groot, High resolution x-ray emission and x-ray absorption spectroscopy, Chem. Rev. 101, 1779 (2001).

[17] A. Carlantuono, X-ray Emission Spectroscopy to retrieve the local spin moment in $3 d$ transition metal compounds, Master's thesis, Politecnioc Di Milano (2017), available at http://hdl.handle.net/10589/133061.

[18] J.-P. Rueff, M. Krisch, Y. Q. Cai, A. Kaprolat, M. Hanfland, M. Lorenzen, C. Masciovecchio, R. Verbeni, and F. Sette, Magnetism and structural $\alpha-\epsilon$ phase transition in Fe monitored by x-ray emission spectroscopy, Phys. Rev. B 60, 14510 (1999).

[19] O. Bunău and Y. Joly, Self-consistent aspects of x-ray absorption calculations, Journal of Physics: Condensed Matter 21, 345501 (2009).

[20] M. Rahman, Y.-z. Nie, and G.-h. Guo, Electronic structures and magnetism of srfeo2 under pressure: A firstprinciples study, Inorganic Chemistry 52, 12529 (2013).

[21] H. J. Xiang, S.-H. Wei, and M.-H. Whangbo, Origin of the structural and magnetic anomalies of the layered compound $\mathrm{srfeO}_{2}$ : A density functional investigation, Phys. Rev. Lett. 100, 167207 (2008).

[22] J. M. Pruneda, J. Íñiguez, E. Canadell, H. Kageyama, and M. Takano, Structural and electronic properties of $\mathrm{SrFeO}_{2}$ from first principles, Phys. Rev. B 78, 115101 (2008). 